\title{
Recent Patents and Designs on Hip Replacement Prostheses
}

\author{
H. Derar* and M. Shahinpoor
}

Department of Mechanical Engineering, University of Maine, Orono, ME, USA

\begin{abstract}
Hip replacement surgery has gone through tremendous evolution since the first procedure in 1840 . In the past five decades the advances that have been made in technology, advanced and smart materials innovations, surgical techniques, robotic surgery and methods of fixations and sterilization, facilitated hip implants that undergo multiple design revolutions seeking the least problematic implants and a longer survivorship. Hip surgery has become a solution for many in need of hip joint remedy and replacement across the globe. Nevertheless, there are still long-term problems that are essential to search and resolve to find the optimum implant. This paper reviews several recent patents on hip replacement surgery. The patents present various designs of prostheses, different materials as well as methods of fixation. Each of the patents presents a new design as a solution to different issues ranging from the longevity of the hip prostheses to discomfort and inconvenience experienced by patients in the long-term.
\end{abstract}

Keywords: Total Hip Replacement/Arthroplasty (THR/THA), Revision Surgery, Stemless Prosthesis, Femoral Head Prosthesis, Acetabular Cup Prosthesis.

\section{INTRODUCTION}

Total Hip Replacement (THR) surgery or Total Hip Arosthroplasty (THA), is a surgery in which the hip joint is replaced with a prosthetic implant. Hip replacement surgery was first performed in 1840 in New York, by Dr. Carnochan who was the first surgeon to insert a wooden piece between the diseased hip joint [1]. In recent years the THR surgery is being considered by most in the field to be a successful elective major procedure especially in restoring mobility to patients. Worldwide, there are approximately one million implants performed annually $[2,3]$. The surgery has become a routine with a minimum rate of early complications, offering patients great relief of pain when medications fail. It further offers tremendous improvement in function and consequently quality of life. Therefore, it is one of the most satisfying operations to both patients as well as surgeons. THR is performed as a solution to several degenerative and traumatic processes which affect the hip joint; it is a reconstructive surgical procedure.

In the past five decades, advances that have been made in technology; innovations in materials, surgical techniques, and methods of fixation and sterilization of prostheses, facilitated hip joint implants go through multiple design evolutions seeking better results and longer survivorship for implants. This resulted in the breakthrough of hip surgery; nevertheless, not all implants were able to withstand the test of longevity. Although the risks of the surgery are low, there are long term complications that contribute to the failure of the prosthesis; the most problematic is the limited life span

*Address correspondence to the author at the Department of Mechanical Engineering, University of Maine, Orono, ME, USA; Cel: (207) 356 5957; Tel: (207) 307 7277; Off: (207) 581 2143; (207) 5812120 ;

E-mail: hind.derar@umit.maine.edu of the prosthesis. Ten percent of the implanted prostheses have a revision surgery within ten years [4]; the average lifespan of the prosthesis is fifteen years $[2,4,5]$, which is a crucial problem to the growing number of young patients under forty years of age. These young patients usually are more active and therefore they will require multiple revision surgeries throughout their life-time. Revision surgery is substantially more difficult than primary THR surgery due to the involvement of removal of existing implant and fixations, complexity of the procedure, and longer anaesthesia and operative time [6]. Preparation of the operating site has to be performed prior to insertion of the new prosthesis with the use of any necessary fixation methods or reinforcement. Revision surgery has a higher rate of intraoperative fractures than primary THR [7]. Postoperatively, patients are prone to have more complications and rehabilitation takes longer. Therefore, it is more risky [5,7], costly and inconvenient to the patient than primary surgery.

It was reported by the Swedish National Hip Arthroplasty Register annual report [7] that between the year 1979 and $2004,73 \%$ of revision surgery was due to aseptic loosening of the implants. "Osteolysis, is defined as the process of progressive destruction of periprosthetic bony tissue" [6], which if not treated could lead to aseptic loosening and failure of the implant. As the femoral head articulates against the acetabular cup, particles of a clinically relative size $(0.1-$ $10 \mu \mathrm{m})$ [4] are released from the implant activating macrophages, consequently, causing osteolysis and resulting in aseptic loosening of the implant leading to the failure of the THA and a necessary revision surgery. The high rates of revision surgery are considered to be affected by prosthetic hip design, materials and fixation methods, as well as the patient's age and activity [8]. To decrease the number of 
revision surgeries, new technical innovations for the prosthesis have been created. This paper presents several patents of various innovations for different components of the prostheses designed to lessen the number of revision surgeries with the intention of increasing the longevity of implants.

The prosthesis typically consists of a femoral stem, a femoral head (ball) that attaches to the stem, an acetabular cup and a fixation agent to secure the stem into the femur and the acetabular cup into the acetabulum in the pelvis, Fig. (1).

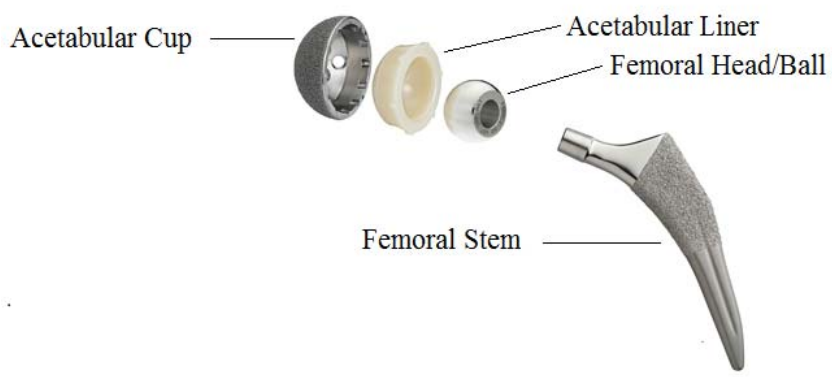

Fig. (1). Hip Joint Prosthesis.

The hip joint is one of the largest joints in the body, similar to a typical ball and socket joint, see Fig. (2), and it is the most weight-bearing. It is located where the femur, the thigh bone, meets the three bones that make up the pelvis; the ilium is at the rear, the ischium at the lower front and the pubis above it [9], as depicted in Fig. (3). These bones congregate to shape the acetabulum (deep socket) at the outer side of the pelvis. At the top of the femur is the femoral head, a ball-like shape, 2/3 of a sphere, it is attached to the femur by the femoral neck which is a short section of bone. The acetabulum forms approximately half a sphere (concaved). A smooth cushion of shiny white articular cartilage, about one quarter of an inch thick, with rubbery consistency, covers the femoral head and the acetabulum. The articular cartilage is made of tough flexible tissues that are kept slippery by the lubricant fluid made in the synovial membrane (joint lining). The synovial fluid is a viscous and sticky lubricant and combined with the articular cartilage they are very slippery, allowing the joint surfaces to move against each other easily without causing damage.

The femoral head and acetabulum are secured in place and prevented from dislocating by large ligaments, tendons and muscles that surround the hip joint (joint capsule).

\section{BACKGROUND ART}

Hip replacement surgery is an old procedure, and one of the most challenging procedures as it plays an important role in helping patients suffering from joint failure due to diseases that include osteoarthritis, avascular necrosis, rheumatoid arthritis, Paget's disease and bone tumors, to name a few [10]. There are three types of hip replacement surgery:

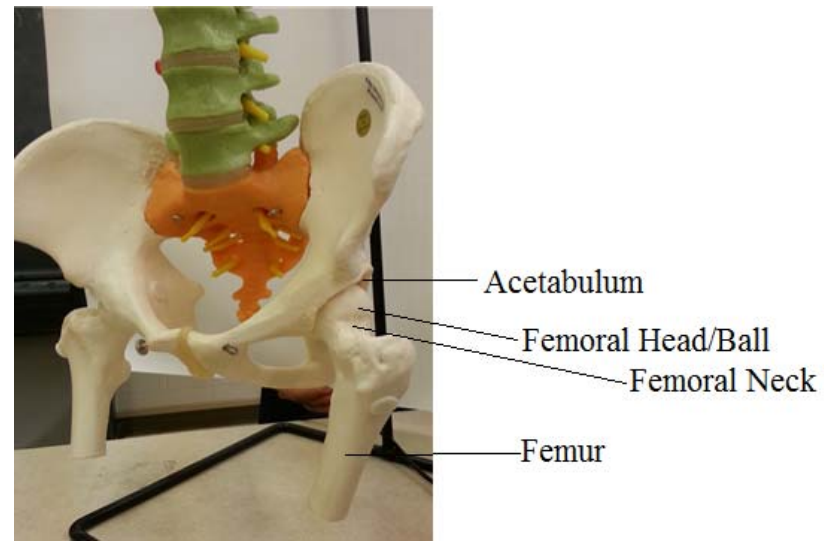

Fig. (2). The hip joint, (picture taken by author from a skeleton in our Biomedical Engineering Lab., in UMaine).

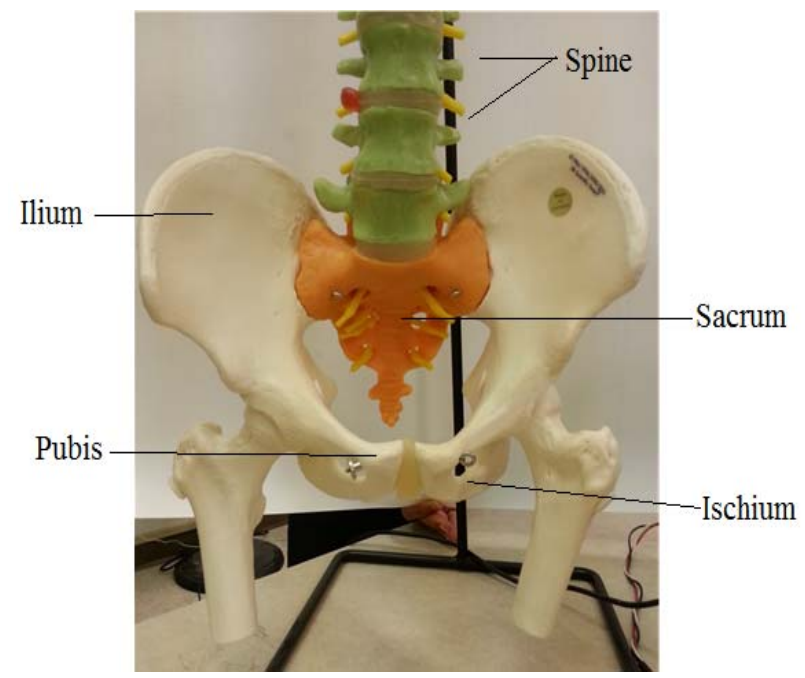

Fig. (3). Bones of the pelvis (picture taken by author from a skeleton in our Biomedical Engineering Lab., UMaine).

1) total hip replacement THR or total hip arthroplasty THA, in which the whole hip joint, ball and socket, is replaced with femoral stem and prosthetic implant

2) partial hip replacement in which only the femoral head is replaced

3) a hip resurfacing procedure in which the socket or acetabulum is replaced and the femoral head is trimmed, reshaped and then covered with a smooth cap.

Some of the complications may be shared by all three types. Thus, not only several surgical approaches have been introduced to find the ideal hip implant, but many aspects have been studied ranging from the novel designs of the various parts of the prosthesis to the different materials employed and their biocompatibility.

The following section reviews a number of recent patents on hip replacement prosthesis. 
U.S. Patent No. 6,248,132 B1, by inventor Charles C. Harris titled "Hip Replacement Prosthesis" [11] and issued June 2001 discusses "Abstract: A hip joint prosthesis including a stem assembly having an outer casing received within the hip socket. The cup assembly outer casing includes threadedly attached upper and lower portions to encapsulate the lining and the lining includes an upper portion having a recess receiving the head and a split lower portion seating the head. The cup assembly also includes an interior spring assembly providing shock absorption between the head and the casing. The stem assembly includes an elongate stem and a cooperating shield attached to the stem, the stem being independently movable following insertion of the stem and shield into the femur."

According to the inventor, an ideal implant should be able to re-produce the mechanism and structure of the original member replaced. It is essential for the femoral head of the implant to be tightly fixed to the stem and that the head is received within the acetabulum. Also, the resulting joint should provide some flexibility or "cushioning".

The inventor [11], presents an improved hip joint replacement over previous arts which surpasses past problems and offers advantages for this invention [11].

The inventor provides a hip prosthesis that delivers a substantial universal joint movement between the stem assembly and the cup assembly. The stem is secured into the femur and the cup assembly receives the femoral head which is received by the prepared hip socket. The socket is being affixed with a tight socket shell. The structural arrangements for the parts provide: rotational movement of the cup assembly about the radial center of the socket and also pivotal movement of the stem about the center of the femoral head while circular movement of the head about the femoral neck axis.

One of the invention aspects is to have the cup assembly consisting of means providing shock absorption between the head and casing.

An additional feature of the invention is that the stem assembly has an extended stem and a shield with means between them allowing the stem to move independently relatively to the shield, after both being inserted into the femur.

According to the inventor [11], the novelty of this invention is to have an implant with the femoral head firmly fixed to the femur and the head "received" within the acetabulum. This invention introduces a less expensive replacement as well as ease of manufacturing and installment. It is considered efficient in providing natural joint movement.

U.S. Patent No. 6,336,941 B1, by inventors; G. V. Subba Rao, Anil K. Goli, titled "Modular Hip Implant With Shock Absorption System" [12] and issued Jan. 2002 introduces "Abstract: A modular hip implant that can be custom fit to an individual patient, including a shock absorption system that absorbs compressive stresses that are imparted to the implant. The size of the femoral ball member, size of the femoral stem, femoral neck length, and tension in the shock absorption system are all individually adjustable parameters, depending on the particular patient. A unique coupling member houses a modular spring mechanism that serves as the shock absorber. The coupling member is received into the ball member to an adjustable depth, the adjustment of which varies the length of the femoral neck. The length of the femoral neck can be adjusted during surgery without requiring additional parts."

The invention [12] presents a device that can absorb compressive stresses conveyed to the implant through the shock absorption system. Additionally, the device may be custom fitted, where the ball and femoral components are selected from a range of ball and femoral members, the selection of the ball depends on the size of the acetabulum socket. This invention [12] also comprises a spring system running through the ball and the neck in order to furnish cushioning between the ball and the femoral stem. The spring is to be chosen from a plurality of springs with different constants and stiffness. The device consisted of two shock absorbers, one attached to the ball and a second attached to the upper part of the femur with a spring between them to cushion the compressive force. This device [12] could be of advantages to the patients as the spring mechanism of contracting and expanding while performing daily activities reduces load bearing, shock and compressive stresses on the implant. This will mean less movement of the implant and hence no loosening resulting in a longer implant life-span. Other advantages of the invention [12] are decreases in complications such as acetabular damage and erosion, and dislocation. Additionally, the spring being custom fitted provides the option of choosing a spring with the right parameters (stiffness). Moreover, the length of the femoral neck can be chosen as required without additional parts since a single coupling member may be installed in the ball to the depth that corresponds to the acquired neck length, this can be adjusted during surgery.

U.S. Patent No. 6,379,390 B1, by inventors, Suresh G. Advani, Michael H. Santare, Freeman Miller, Makarand Joshi, and issued April 2002, introduces "Stemless Hip Prosthesis," [13], in which "Abstract: A stemless hip prosthesis uses one or more cables which wrap around the prosthesis and the femur to mount the prosthesis in place thereby avoiding the need to provide the prosthesis with a stem located in the medullary canal."

According to the inventors; one of the main reasons of THR failure is bone resorption which might be due to stress shielding of bone as a result of a stiff prosthesis being used, plus extra shear stresses where both prosthesis and bone contact. In the classical surgery, the current designs of prostheses use a stem that is inserted in the femur. Therefore, the inventors are introducing a stemless hip implant. To divert the load from being transferred through the stem of the prosthesis the load is applied at the proximal end of the femur. To apply the bending moment through the cross section of the femur with the greater trochanter included would acquire a new fixation technique that decreases the interface shear stresses, relative torsion and stress shielding. Since cables support axial loads, they do not increase the bending stiffness of the bone. The short flexible stem bolt arrangement in the medial calcar area reaches into the femur offering torsional support and fixation, while increasing local interface shear stresses. The cables contacting the tendons 
increase slightly the local stresses, permitting a "natural" stress spreading through the proximal femur cross section. Hence, stress shielding is at a minimum because the effective bending of the femur is not increased. The notion of this design is to eliminate the "interface shear stresses" by the use of familiar surgical high strength steel cables for fixation, wrapping them around a portion of the femur and prosthesis. The cables uphold axial loads and therefore do not raise the bending stiffness of the bone. Moreover, the inventors [13] believe that this design will simplify revision surgery if needed as it would necessitate the cables being tighter, which is an important feature. The cables and fixture screws are to affix the prosthesis tighter without the use of a stem inserted into the femur.

U.S. Patent No. 6,425,922 B1, by inventors; Bill J. Pope, Jeffrey K. Taylor, Richard H. Dixon, Clayton F. Gardinier, Louis M. Pope, Dean C. Blackburn, Michael A. Vail, Kenneth M. Jensen, and issued July 2002, titled " Prosthetic Hip Joint Having At Least One Sintered Polycrystalline Diamond Compact Articulation Surface," [14] describes "Abstract: Prosthetic joints, components for prosthetic joints, super hard bearing and articulation surfaces, diamond bearing and articulation surfaces, substrate surface topographical features, materials for making joints, bearing and articulation surfaces, and methods for manufacturing and finishing the same, and related information are disclosed, including a prosthetic hip joint having polycrystalline diamond articulation surfaces and at least one sintered polycrystalline diamond articulation surface."

This invention is targeting a minimum wear and no debris and therefore longer life-span of prosthesis with expectancy of life-long patient use. Diamonds of various types and super-hard materials are used in this invention in the bearing surfaces because of their low coefficient of friction and resistance to wear, and if particles due to wear are created they will be of no significance. Polycrystalline diamond compacts or super-hard materials are to form one of the articulating surfaces of the implant. This will reduce the incidence of osteolysis which is a major advantage.

US Patent No. 7,641,699 B2 by inventor Anthony S. Unger, titled "Femoral Head Calcar Loading Prosthesis," [15] and issued January 2010 describes "Abstract: Provided is a novel hip replacement implant system that includes a ball assembly having a ball trunnion, the ball assembly being configured as a replacement for the head of the femur and capable of being secured by the ball assembly trunnion to a calcar implant element that can be securely seated in the calcar bone of the femoral neck, thus maintaining the load transfer function of the calcar bone of the femoral neck. A method of using the device is also provided."

Most of the conventional hip replacements include a design of a stem inserted in the femur canal replacing a proximal portion of the femur. This places unnatural stresses on the femur and consequently pain and limitation to the patient's activities. Moreover, the technique used in removing the neck of the femur may cause destruction of the calcar bone and eventually exposes the patient to bone weakness and fractures that could lead to revision surgeries.
This invention presents a solution to the problems of typical hip implants, and provides techniques to replace the conventional one by reproducing the hip joint articulation aspects and also utilizing the natural load transferring femur calcar bone to prevent prosthesis failure and revision surgery. Thus, the inventors [15] present a novel hip implant system comprised of a ball assembly having a ball trunnion or trunnion to facilitate the capability of the ball being affixed to a calcar implant element which can be securely positioned in the calcar bone of the femoral neck and articulating with the ball assembly. Also, included is an acetabular component that is formed and inserted in the hip and articulated with the ball assembly. The ball assembly is of metal as well as the acetabular components; the metal ball is to replace the femoral head and the acetabular component is measured and designed to be inserted in the hip pelvic cavity and articulating with the metal ball component. Additionally, the invention provides a procedure of embedding the hip replacement prosthesis implementing minimally invasive surgery to implant the prosthesis.

U.S. Patent No. 7,833,277 B2, by inventors; Joseph Saladino, Mark E. Nadzadi, Brian Burkinshaw, titled "Femoral Head Assembly With Variable Offset," [16] and issued Nov. 2010, presents "Abstract: A proximal femoral ball assembly having a variable offset that is selectively adjustable to conform to various anatomical conditions encountered during a femoral surgical procedure. The femoral ball assembly generally includes a head, a neck, and an adjustment mechanism. The head has a smooth spherical outer surface that is adapted to engage an acetabular component or native acetabulum. The neck extends outward from the head and removeably connects to the head using a threaded attachment."

This invention relates to orthopedic implant prostheses for THA and more specifically to a proximal femoral head assembly of variable offset and selectively adjustable to adapt to any anatomical conditions during surgery.

A hip joint implant typically may consist of a femoral stem, a socket or acetabulum, and a femoral head or ball. During surgery, the femur is prepared to receive the implant femoral stem which is then inserted into the intramedullary canal of the femur to the proper depth and orientation, while the appropriate ball is fixed to the proximal end of the stem. The ball is fit into the socket or acetabulum where it facilitates an articulation surface between the femoral prosthesis and acetabulum. Between the femoral head and stem there is a neck or trunnion connected to the stem at one end and in some designs connected to the head, and it is usually of cylindrical shape.

To ensure proper functioning of the prosthesis of this invention [16] after implanting, several crucial features are to be considered. One of these features is the femoral head "offset".

According to the inventors, "femoral head offset is the horizontal distance from the center of rotation of the femoral head to a line bisecting the long axis of the femur from a standing A-P x-ray." The offset of the proximal femoral component is similarly the horizontal distance between the center of rotation of the ball and the stem's long axis. The 
amount of offset between the ball and the stem implemented by the surgeon during surgery significantly affects the performance and success of the prosthesis. If the patient's natural anatomical needs are not met by the offset, then the prosthesis can be positioned either too far laterally or medially.

Additionally, a decrease in femoral offset medially results in shifts or moves of the femur closer to the pelvis and it can cause in some patients impingement of the prosthesis as well as loosening or laxity of local tissue resulting in implant instability, subluxation and dislocation. A further disadvantage of the offset decrease is that as the abductor muscles balance the pelvis they employ larger force generating discrepancy that might cause a patient's limp, and there is greater polyethylene wear between the ball and the acetabular components.

An increase in the offset laterally on the other hand causes the femur to move or shift further than the pelvis. An increase in offset is sometimes desirable for various reasons such as:

\section{1. may lessen the impingement risk}

2. may improve the tension of soft tissues and hence the implant will have more stability

3. the abductor muscles can be appropriately balanced and the patient gait improves, which also results in lesser wear and loosening over time.

Due to the aforementioned disadvantages, hip prostheses are marketed with different offsets. Hence a typical system may have a number of different offsets for each femoral head and therefore the manufacturer will need to have an inventory ranging between 18-30 different femoral heads, which is costly for maintaining and distributing.

Therefore, for the above mentioned disadvantages and others, the inventors [16] present a proximal femoral ball assembly comprised of a head, a neck, and an adjustment mechanism. The ball is of a smooth spherical outer surface to interact smoothly with the acetabular component or the original acetabulum. Further, the neck is extended outwards from the ball and detachably connects with the head by threaded connection.

An additional advantage of the invention is that it furnishes a plurality of small increments of femoral offsets as small as $1 \mathrm{~mm}$ increments, and these offsets can range between $-10 \mathrm{~mm}$ to $+10 \mathrm{~mm}$ while the inventors think of the possibility of a range up to $+30 \mathrm{~mm}$. This enables appropriate matching of the patient's anatomical needs.

In another embodiment of [16], a femoral ball system with different sized heads and spacers are provided that can be employed with a single neck offering several femoral offsets with plurality of various sized spherical balls.

In another embodiment of the above invention [16], two parallel axes that form an acute angle with longitudinal axis of the stem, extend through the femoral ball assembly. The first axis or central axis is concentric with the spherical head body while the second axis or eccentric axis is concentric with the threaded head bore and also concentric with the adjustment mechanism and neck of the femoral head assembly.

Another advantage of the invention is the eccentric neck (or the offset from the femoral head) presents an increased array of movement when attached to the stem, thus the increase replicates the hip motion and stabilizes the prosthesis. Additionally, the eccentric neck presents a femoral prosthesis which will not be prone to impingement, subluxation or dislocation.

U.S. Patent No. 7,931,691 B2; by inventors; Xue Li, Ping Xie, Kerry Y. Xie, titled "External Proximal Femoral Prosthesis For Total Hip Arthroplasty," [17] and issued April 2011 presents "Abstract: A external femoral component for mechanically housing onto exterior remnant of natural femur and coupling with articulation elements in order to use in total hip arthroplasty defines as a hollow shell comprised of asymmetrically bell shaped cup, at least one rigid elongated stem downwardly extended from the lower rim of the cup and a plurality of an cylindrical object upwardly protruded from top of the cup for pairing with articulation elements and coupling with anchoring means and the tension anchoring means fixes the hollow shell on the bone surface through an interlocking mechanisms."

The invention introduces a femoral prosthesis and in particular relating to external proximal femoral element seated in the outside area of the rest of the proximal femur, affixed securely with installation by tension means.

According to the inventors [17] there are disadvantages to the conventional prostheses that have been used in total hip replacement and which comprise stem designs. These disadvantages include:

- The applied bending moment through the stem causes stress concentration at the proximal, medial and the distal lateral ends of the prosthesis, respectively, i.e. in a comparatively small area to the large force applied to the area of the original femoral head.

- High shear stresses result from the transfer of axial loads and torsional moments to the bone through the boneprosthesis interface.

- Reduced bending displacements because of the prosthesis high stiffness results in stress shielding between the bone and prosthesis. The discrepancy in stiffness adds to the interface shear stresses.

- Bone features are reduced as a result of insufficient blood circulation at the implant site.

Accordingly, the prostheses with stems may not exist long in the body due to the above reasons, and therefore these prostheses are best for patients over 60 years of age.

Additionally, implanted prostheses generally may cause proximal femoral resorption due to the following:

- Stress shielding under the implant,

- No biocompatibility initially between bone and prostheses causing increased resorption,

- Insufficient femoral head vascularity that leads to osteonecrosis. 
The aim of these inventors [17] is to present a femoral component without the need of removing all the femoral neck, and without the insert of the prosthetic stem into the medullar bone of the femur. Using the proposed technique of installing, the prosthesis will spare damages to the intramedullar femoral canal and bone marrow of proximal femur. Hence, bone growth will not seize.

The inventors [17] also aim to introduce a locking technique for the femoral prosthesis. The advantage of this invention [17] is that it offers the patient a quick recovery and considerable mobility after surgery due to the following reasons:

- The simplicity of the surgical procedure allows it to be performed in short time with the least bone damage.

- The multiple mechanical and biological fastenings used in the prosthesis provide an improved interface between prosthesis and the rest of the femur.

Moreover, the inventors [17] claim that this type of femoral prosthesis is easy to repair, remove or replace by a new one of the same type or a stem one if failure occurs later on. Also, its longevity will extend and therefore will be more promising to younger patients.

U.S. Patent No.7, 947,084 B2, by inventor; Helmut D. Link, titled "Hip Joint Prosthesis With A Shaft To Be Inserted Into The Femur," [18] and issued May, 2011, presents "Abstract: A hip-joint prosthesis includes a shaft which is configured to be inserted into the femur and whose surface has an osteoinductive finish. This finish is provided exclusively in the metaphyseal portion of the shaft and laterally from the line delineating the maximum anteroposterior dimension of the shaft cross section. This ensures a better involvement of the metaphyseal spongiosa in the flow of forces, without compromising the ability to perform follow-up surgery on the prosthesis."

The goal of the inventor [18] here is an improved fixation method of the femoral prosthesis in the bone without a compromise in the ability of performing a follow up surgery.

The hip prosthesis in this invention is comprised of a head and a neck that has a shaft to be inserted in the femur. Osteoinductive coating for hip prosthesis with a shaft is performed exclusively on the metaphysical portion where the shaft is placed laterally from the line of maximum anteroposterior (AP) dimension.

The surface area to be finished with osteoinductive substance is defined according to this invention in a different manner than previous arts. The osteoinductive finish is applied at the part of the metaphysical portion where many surface areas have directional normal with lateral components. According to previous experience these surface areas are not expected to have any connection to the bone permitting transmission of tensile forces, while this connection facilitates it to the most part. The earlier application (PCT/EP03/05292 not belonging to the published prior art) proposes arranging the coating on surface areas pointing in the medial direction, the opposite of this invention [18]. It is an advantage that the material can be coated with any type of coating and can be porous.
Immediately after the surgery the effect is that the bone cells quickly develop close and in contact with the prosthetic surface, consequently neither a gap nor a layer of intermediate connective tissue is formed due to the relative movement between the bone and the bone surface, thus intimate connection is more difficult or impossible. The invention [18] provides more rapid accumulation of bone on the trochanteric surface of the prosthesis as well as fast incorporation of bone into the pores resulting in quick permanent fixation of the trochanteric area of the bone to the prosthesis and is thus involved in transmission of forces. In opposition, the connection to the bone on the other surface areas is attained only to the prior anticipated extent. The area outside the trochanteric is ready to access and is nonproblematic in a follow up surgery if required. Although the bone might be tightly bound, therefore the physician finds the exact conditions he/she is familiar with when performing follow up surgery. According to the invention, the surface area where the osteoinductive substance consists of pores or undercuts relative to the lateral direction, and the bone substance due to the osteoinduction can both cohere to the surface and anchor firmly onto it.

Portions of the osteoinductively finished surface areas of the prosthesis, which point in the ventral and dorsal directions, are preferably placed at a certain distance closer to the cortex and farther from the mid-plane of the trochanteric bone where it is sometimes less dense than near the cortex. Hence, these surface areas in the AP direction should be not too thin. It is favorable for the thickness and thus distance between dorsal and ventral surface areas to be over $6 \mathrm{~mm}$ but more beneficially between 9 and $15 \mathrm{~mm}$ according to [18]. Press-fitting of the surface areas can promote growth of new bone cells onto the prosthesis surface and it is beneficial if these areas and their mating surfaces are made of wedge shape in the direction of insertion into the bone. Also, the rasp used to prepare the receiving site for the prosthesis shaft insertion, is of a smaller cross-sectional dimensions. Thus, when the shaft is inserted in the femur these surface areas dislocate bone substance.

U.S. Patent No.8, 133,284 B2, by inventors; K. Scott Ely, Ashok C. Khandkar, Ramaswamy Lakshminarayanan, Aaron A. Hofmann, titled "Hip Prosthesis With Monoblock Ceramic Acetabular Cup," [19] and issued March 2012, introduces "Abstract: An improved hip prosthesis includes an acetabular cup bearing component constructed from a relatively hard and high strength ceramic material for articulation with a ball-shaped femoral head component which may be constructed from a compatible ceramic or metal material. In one form, the acetabular cup further includes a ceramic porous bone ingrowth surface adhered thereto for secure ingrowth attachment to natural patient bone."

Generally hip prostheses are comprised of femoral head or ball-like component, a hip stem component and an acetabular component. The acetabular cup is to receive the femoral head, and the femoral head articulates relatively to the acetabular cup component. Between the acetabular cup and the femoral head, a polymer based bearing insert usually of high density or high molecular weight polyethylene (PE) 
or similar material is affixed in between the cup and the femoral head for smooth articulation securing low-wear articulation. Studies have illustrated that these polymeric bearing inserts produce substantial wear debris in the long term and may also be partially a contributor to osteolysis [2, 6] and bone resorption and thus prostheses failure. The inventors [19] have introduced an improved hip prosthesis that includes improved implantable and biocompatible materials to achieve ultra-low wear using ceramic-onceramic or a ceramic-on-metal articulatory interface. Thus, they propose to eliminate the use of the customary polymerbased bearing insert and the wear debris problems resulting in longer longevity for the prosthesis. Moreover, obtaining a cup with all-round thinner diameter that facilitates the use of a larger head for patients with small bones. Another aspect of the invention [19] is a unipolar prosthesis that consists of a ceramic acetabular cup of a shell shape or cup shape geometry outlining to some extent a low porous substrate combined with a surface of a higher porous bone ingrowth. This higher porous surface extends over the acetabular cup upper surface to be tightly fixed in the prepared acetabulum by bone ingrowth. The low porous substrate defines a partial spherical cavity to seat and articulate with the femoral head. The pores size range between 100 and 500 microns. The head is made of compatible and extremely strong and tough ceramic material or metal material for instance, a biocompatible cobalt chrome alloy or similar.

The ceramic material favoured for the acetabular cup consists of both high flexural strength and high "fracture toughness" material as doped silicon nitride $\left(\mathrm{Si}_{3} \mathrm{~N}_{4}\right)$, with flexural strength ranging between $700 \mathrm{MPa}$ and $1000 \mathrm{MPa}$ and relatively high fracture toughness ranging between 7 and 10.5 Mega-Pascal [19] for ultra-low wear and a reduced risk of brittle fracture. The unipolar prosthesis is comprised of acetabular cup with a relatively low porosity substrate combined with a higher porosity bone ingrowth surface. The pores size range between 100 and 500 microns.

U.S. Patent No. 8,323,346 B2, by inventor, Slobodan Tepic, titled "Wear-Reducing Geometry of Articulations in Total Joint Replacements," [20] and issued Dec. 2012 discusses "Abstract: The invention reduces wear in total joint articulations by modifications of the shape of either component of the kinematic pair, so as to result in an annular surface contact between the two components. Fluid trapped between the two components within the inner contour of the annular contact area is pressurized under load due to elastic deformation of the components and exuded out through inter-articular gap over the surface of contact, aiding in lubrication and reducing the wear. Reduced to practice for a total hip joint with UHMWPE-metal pair, the wear rate tested in a hip joint simulator up to five million cycles was reduced by factor seven to fifteen compared to conventionally shaped components."

The invention [20] addresses a solution to the most contributing factor to aseptic loosening: wear of Ultra High Molecular Weight Polyethylene (UHMWPE) which leads to shorter lifespan of artificial joints. The notion is to reduce the wear primarily and hence increase the "overall robustness of the joint and possibly relaxing the need for the exacting production technologies."
Through changes in the geometry of the articulating surfaces, a decrease of the contact stresses as well as an improvement in the lubrication of the area of contact will result, and therefore the wear characteristics of the implant is improved. According to this invention [20] the presented changes in the geometry are a combination of either a spherical head with an aspherical cup or an aspherical head in a spherical cup. Note that both assemblies should have a theoretical surface contact over a band that is centered approximately 45 degrees with respect to the revolutionary axis.

U.S. Patent No. 8,372,155 B2, by inventors; Michael Antony Tuke, Andrew Clive Taylor, titled "Acetabular Cup Prosthesis And Method Of Forming The Prosthesis," [21] and issued Feb. 2013, introduces "Abstract: An acetabular cup prosthesis comprising an acetabular cup having a rim and comprising a metal band applied around the outer circumference of the acetabular cup prosthesis and adjacent to said rim."

This invention [21] presents an acetabular cup having a rim and a D-shaped metal band applied around the cup outer circumference and adjacent to the rim. The metal band provides additional strength to the prosthesis plus the hoop compression level required. The acetabular cup may be made of ceramic or of a material with similar ceramic properties such as strength, stiffness and rigidity. An advantage to this invention [21] is that it improves resistance to fracture. Further, the metal band helps to pre-stress the ceramic, where there is a ceramic cup or ceramic liner, since nonstressed ceramic liners while in use are liable to fracture as in previous arts.

The band in this invention [21] is made of any suitable metal, favourably Titanium as well as Cobalt/Chromium. On the outer surface of the band there are ribs extending from the cup, these ribs when in use will facilitate the required torsional stability while interacting with the pelvis. In one arrangement, around the circumference of the band, favourably three ribs may be placed at a plurality of points, or located at three equally spaced positions. The ribs configuration may be the same or differ wherever there is more than one.

The band in this invention [21] can be set on its outer surface to stimulate bone integration, or another aspect is to coat the outer surface with a substance that stimulates bone growth such as hydroxyapatite. The same concept can be performed for the shell. If a Titanium shell is used, the thickness of the Titanium should range between $1 \mathrm{~mm}$ to 3 mm.

According to this invention [21] the ceramic cup and liner can be made of any material that is biocompatible, hard and wear resistant with thickness ranging from 2 to $5 \mathrm{~mm}$. The ceramic materials to be used may be silicon nitride or doped silicon nitride, to name a few.

The band thickness will usually be of a few millimeters, and its surface that connects with the cup is usually flat and its outer surface may be curved so the cross-section may be of a D-shape. The thickness at the thickest point may be of 1 to $3 \mathrm{~mm}$ thick. 
There are various techniques to apply the band to the acetabular cup [21]. One of these is to press-fit it, but this might be difficult since the band is a tight fit on the cup. Therefore, one method is to heat the band to fit appropriately. The heating may be by induction which is advantageous as it only affects the metal band and the ceramic cup stays unheated. Depending on the temperature that heats up the band a load may be required for pressfitting. Heating may range from 500 to 700 degrees $C$ while the press-fitting load may be around $5 \mathrm{kN}$ [21].

An additional advantage of this invention [21] is that the acetabular cup can withstand any forces while inserting a prosthesis into the acetabulum without damaging or distorting or separating the band. The local compression at the rim of the liner causes a decrease in the diameter. Nonetheless, the spherical shape of the prosthesis is considerably the same.

U.S. Patent No. 8,470,049 B2. By inventors; Bradley Walter, Declan Brazil, Timothy McTighe, titled "Neck Sparing Total Hip Implant System," [22] and issued, Jun. 2013, discusses "Abstract: A femoral prosthesis. The femoral prosthesis includes an implant body having a proximal end a distal end a shoulder at the proximal end, the shoulder being structured and dimensioned for a tight press fit into the neck of a femur. The implant body includes a trunk at the distal end, the trunk having a wedge formed by a tapered portion extending in the direction of the distal end of the implant body. The implant body also includes a medial column extending from the shoulder toward the distal end. The wedge, the medial column, and the lateral column are to provide multi-planar stability for the implant body and surface area for fixation of the implant body."

The disclosure [22] is relating to femoral prosthesis in particular and femoral neck sparing implant. To accomplish implant stability and longevity, a lot of bone loss is involved a problem that was attended to through the years by research and studies and hence tissue sparing plus bone sparing surgical techniques were developed. Bone loss is due mainly to osteolysis and stress shielding. Through improvement of wear properties of modern bearing surfaces loss of bone due to osteolysis was largely decreased. Bone loss due to stress shielding was addressed by "loading the proximal femur with tapered stem geometries or surface replacement devices." These devices have their limitations such as: the procedure requirement of larger exposure, in other words no tissue sparing. Also, there is the possibility of early or late collapse due to the poor foundation of the femoral head, and the likelihood of fracture of femoral neck. Thus, the inventors [22] are minimizing these problems by their invention.

U.S. Patent No. 8,506,644 B1, by inventors; MarieChristine Ho Ba Tho, Francois Roux, Maximilien Vanleene, titled "Acetabular Prosthesis To Be Fixed Without Cement," [23] and issued Aug. 2013, introduces "Abstract: The invention relates to an acetabular prosthesis comprising an, in general, hemispherically shaped insert (1) having a crown (5) and an apex (4), this insert being provided, on its outer surface (2), With at least one fin. The invention is characterized in that the fin (6) has a face (9) starting from the crown (5) of the insert (1) and extending toward the apex
(4) of the insert while forming a crest (10). This crest (10) has an, in particular, curved shape."

The invention [23] relates to fixing the acetabular prostheses without cement. The total hip prostheses comprises a femoral prosthesis which includes a stem provided with a femoral head, the stem is to be inserted in the femur, and an acetabular prosthesis to be fixed in the acetabular cavity which receives the femoral head. There are various methods of fixing the acetabular prostheses to the bone such as cemented or uncemented. Note that both methods could be efficient but each has its advantages and disadvantages. The cemented technique which is usually based on PolyMethylMethAcrylate (PMMA) is good in the long-term. However, aseptic loosening frequently results [23]. Another means of fixing without cement is by mechanical means, thus the acetabular prosthesis few months post implantation would be able to have effective mechanical strength of the prosthesis in the bone to stimulate osteointegration or integration of the prosthesis to the bone tissues. Cementless prostheses may consist of a metal cup and a PolyEthylene (PE) insert positioned in the metal cup which is fixed in the bone by pads, screws, fins or pins, also the acetabular implant may be fixed in the bone cavity by what is known as press fit or forced insertion [23]. The invention [23] is proposing to fix the acetabular prosthesis without cement, (as mentioned in the abstract). Introducing the facet may be advantageous as it may distribute the exerted stresses by the insert over the cortical bone, and also the crest of the fin being of a curved shape so the prosthesis is placed in the cancellous bone to avoid damage to the bone tissue. The fins fix the insert in place as the femoral head move and they also provide "mechanical strength of the prosthesis in the bone until osteointegration is sufficient to take over."

In the invention [23] the prosthesis external convex surface has a roughness of $3 \mu \mathrm{m}$, and is covered with a layer of hydroxyapatite coating, hence facilitating colonization by bone cells and consequently stimulating osteointegration of prosthesis.

Also, the internal surface of the insert, which will be in contact with the femoral head, is coated with Diamond-Like -Carbon (DLC) which consists of large amount of "sp ${ }^{3}$ hybrids" that gives it similar properties to diamonds. Using this coating increases the wear resistance properties. Further, the DLC coating has very good biocompatibility.

U.S. Patent No. 8,603,182 B2, by inventors; Richard Lambert, Terry McLean, David Kelman, titled "Hip Prostheses," [24] and issued Dec. 2013 and presents "Abstract: Bipolar prostheses which include various structures and other techniques for optimizing material wear and mechanical strength properties. Such prostheses feature, for example, improved resistance to polyethylene wear while also reducing potential for dislocation of the femoral stem from the prosthesis. Such techniques and structures include varying wear resistance and mechanical strength treatment in various components of the prostheses or portions of those components as desired to improve, accentuate or optimize wear performance and dislocation reduction, locking ring structural features, structures for retaining locking rings in the bipolar prosthesis shell, and structures for limiting or 
reducing movement or rotation of locking rings and liners in bipolar prosthesis shells."

Bipolar prostheses usually offer two bearing surfaces that are between the femoral head and the acetabulum. The first bearing surface; the shell where its outer surface is usually smooth and made of various materials such as metal, ceramic or other materials. The inner bearing surface is the liner which is mostly made of polyethylene material such as Ultra High Molecular Weight Polyethylene or UHMWP. The liner is to fit securely into the shell where it receives the femoral head and provides smooth articulating movement between the acetabulum and the outer surface of the shell and the relative movement between the femoral head and acetabulum. The two bearing surfaces will absorb the articulation movement and therefore lessen protrusion and erosion of the acetabulum. Thus, in [24] the shear forces are distributed between the inner and outer bearings. The inner bearing also absorbs some of the movement between the acetabular and the femoral head; hence the acetabular wear is subsided. Bipolar or dual bearing surface facilitates more array of motion than unipolar designs or conventional THA.

According to the inventors [24], although there are advantages to the bipolar prostheses but there are disadvantages such as; the possibility for dislocation of femoral head relative to the shell and liner. Further, the polyethylene wear is an additional problem. These issues are also a concern for acetabular components as well as prostheses for other joints. Dislocation is a serious problem since usually it requires revision surgery. Various hip prostheses and bipolar designs were invented to solve this issue. Some designs involved retaining or locking rings. Overemphasis of prevention of dislocation can result in reduction of motion between the femoral stem and the shell/liner. Moreover, other designs addressed this problem by introducing a split in the annulus formed by the locking ring. Nevertheless, these split rings still have problems such as contracting and expanding of the diameter after installation which may lead to polyethylene wear according to [24].

Polyethylene wear is known as one of the major problems to hip prostheses. Several methods have been undertaken to reduce polyethylene wear such as specialized treating of polyethylene, metal and other surfaces. Further processes were enhancements of polyethylene wear performance for instance irradiation, plus procedures that will increase the cross-linking or features of other materials in polyethylene. Although this is of benefit, it has an effect on the polyethylene mechanical and physical characteristics, e.g., yield strength, tensile, elongation and impact strength as it reduces these characteristics. Polyethylene liners and locking rings are also affected in the manner that they are unable to hold the femoral stem head and avoid dislocation.

To resolve the aforementioned issues the inventors in [24] present several techniques and structures to reduce polyethylene wear while neither compromising nor lessening the ability of the shell/liner in preventing dislocation of the femoral stem head. They also introduce a change in the material properties of parts of the shell/liner, shell and locking ring which are at pre-set locations, by treating the material at these sites to "emphasize wear performance." On the other hand, other sites can be also changed or unchanged or not treated to highlight features as yield strength, tensile strength, elongation and impact strength among others.

The said invention [24] exploits optimum performance properties of various types of irradiated polyethylene materials to exhibit improved wear performance when placed in articulating and non-articulating relationships with metal and other surfaces, certain structures and processes to optimize cross linking of various regions of the liner which are in a position to accept increased load from the femoral stem head.

U.S. Patent No. 8,679,188 B2, by inventors; Jeffrey J. Shea, Vincent W. Shotton, David C. Kelman, titled "Universal Liner," [25] and issued March 2014, introduces "Abstract: The present invention relates to universal liner assemblies for use during hip joint replacement surgeries. The universal liners allow the surgeon a greater degree of selection of liners and shells, without being tied to typical liner/shell connections based on material connection constraints."

According to [25], when the surgeon is making her/his choice of implant she/he takes under consideration several factors such as age, weight, gender, level of activity and approach of surgery, among others. Then there are factors concerning the implant itself such as design, materials used, and methods of fixation. There is also the choice of the liner and shell combined as they both are material and design specific. For instance they could be ceramic liner and metal shell or metal on metal, or Polyethylene liner and metal shell. The ceramic liner metal shell uses for connection what is known as Morse taper connection [25] where the tapered outer surface of the liner and the taper on the inner surface of the shell join. The tapers secure the connection and so the liner locks in the shell. On the other hand, when the liner is made of polyethylene and the shell is made of metal, this may not happen because the polyethylene has a low push-out force resistance and so the liner tends to "push" outwards of the shell as polyethylene warms up due to the body temperature and therefore the liner tends to pop out. Thus, there are other types of locking connections for polyethylene-metal components such as axial locking and rotational locking features. Sometimes, the surgeon chooses to use a shell and a liner independent of each other that might be of benefit to the patient. Hence to secure their connection some surgeons used cement, nonetheless there is no available system to furnish this flexibility.

Hip implants have a limited longevity mostly due to the wear debris caused by the articulation movement between the various parts of the implant. Therefore, it would be advantageous to have a universal liner that "can cooperate with a currently-implanted shell" to reduce shell removal trauma on the patient [25].

This invention [25] embraces several embodiments of the universal liner assemblies. The invention also includes different kits of a variety of universal liners in addition to techniques of manufacturing and implanting.

Universal liners would provide the surgeon with the choice of various liner-shell connections prior to surgery. Another advantage is that the components of implants can be 
exchanged, e.g. liners of several materials used with different kinds of shells. Thus, it is advantageous to the surgeon in a revision surgery to have the variety of materials that can be used with an existing shell. Additional advantage of the invention [25], the band assembly and the liner component are assembled as one piece to form a universal liner.

U.S. Patent No. 8,700,198 B2, by inventors; Justin Steve Conway, Ryan L. Landon, Jeffrey Joel Shea, and titled "Implant Components and Methods," [26] and issued April 2014, presents "Abstract: Systems, devices, and methods are provided for orthopedic implants. The implants may include a base member, such as an acetabular shell or an augment that is configured to couple with; an augment, flange cup, mounting member, or any other suitable orthopedic attachment. Any of the implantable components may include one or more porous surfaces. The porous surface may be textured by protrusions that connect to and extend from the surface. The sizes and concentration of the protrusions may be varied for specific applications to accommodate different implants and patient anatomies. A porous implant may also include one or more internal or external solid portions that strengthen the implant."

According to the inventors [26] when a hip joint is affected by degenerative disease or other reasons and requires replacement, often the prostheses replacing the original joint comprise two bearing surfaces between the femoral head and the acetabulum. The first is a prosthesis shell or acetabular cup where the second is a liner. The two bearings vary in their materials according to inventors. The liner is usually fitted securely within the shell offering inner bearing surface to receive the prosthetic femoral head, and allow a smooth articulating movement between the femur and the acetabulum.

According to the inventors [26], there are problems and shortcomings with previous arts and to overcome these issues they present systems, devices and methods for modular implants, besides improved features such as bone screws, coatings, and various augment removal and insertion options for a modular implant. The implants may consist of a base member as an acetabular shell or a configured augment to connect with an augment, flange cup, mounting member or any appropriate connection. Furthermore, the connections may be capable of adjusting about the base member. Several techniques of affixation are used between the components of the implant. In other arrangements there are surface characteristics which will facilitate friction permitting ingrowth of bone between the implants and the patient's bone regardless of the bone quality.

- An example of the invention: an implant device consists of a surface that connects to the joint of the patient. The implant may have at its surface spread pores. The protrusions at the surface may be of any form and of any density, also may expand to any appropriate height ranging between about $50 \mu \mathrm{m}$ and about $2000 \mu \mathrm{m}$. The optimum surface will be with protrusions that are adequately large to produce the appropriate friction between the bone and implant, but at the same time not too small to permit a high degree of bone ingrowth through the porous surface.
- Another example: a porous implant may comprise internal or external "strengthening ribs" to support adjacent porous structures.

- The systems, methods, and devices described in the invention [26], having both a profiled macrostructure and a porous microstructure, are beneficial to an orthopedic surgeon as they can allow utilizing customizable, "patient-specific" implant.

\section{CONCLUSION}

There have been numerous fairly recent inventions addressing hip prostheses. Nevertheless, there are still problems that affect prostheses functions and longevity. In this paper a few selected patents that have addressed various problems associated with the design of different parts of the hip prostheses are presented. These patents also introduce a number of design novelties that are discussed in this paper.

The patents chosen for this paper were of special interest as each discussed a different significant issue whether in design, materials or fixation methodologies and presented a set of diverse solutions. For instance:

- Introducing solutions to femoral stem implants and their consequent complications due to insertion of a stem into the excavated femur. Thus to overcome the disadvantages, stemless implants were presented and with different methods of fixation. Although, these designs may be beneficial but they may still encounter installation and stability issues in early stages. Further studies need to be performed.

- Introducing shock absorption systems with adjustable parameters such as size of femoral ball, stem and neck length, where each could be individually adjusted.

- To reduce the problem of wear debris generation, various approaches were addressed such as: wearreducing geometry of articulation. Also, using different materials for coating since coating increases the wear resistance features, such as in DLC coating which has very good compatibility. The materials can also be porous.

- Use strong material such as diamonds, which are known for their low coefficient of friction and resistance to wear, to minimize wear debris during motion and hence extend the implant survivorship. The material is to form one of the articulating surfaces.

- Implants that spared the removal of the femoral neck and without insertion of stem in the medullar bone with locking techniques for femoral prosthesis. These implants will lead to quick recovery and considerable mobility after surgery.

- Introducing a design that spares the calcar bone and the method of using the device.

- Femoral head assembly with variable offset that is selectively adjusted.

Each patent focuses on a different problem area of the hip prostheses, making a simple comparison of the patents unhelpful. However, even though the patents differ in the approach with which each addresses the problem of hip 
failure all have the same objective to obtain the optimum implant with the extended life-span for the patient and possibly no revision surgery.

\section{REFERENCES}

[1] J.V. Bono, J.C. McCarthy, T.S. Thornhill, B.E. Bierbaum, and R.H. Turner, Eds, Revision Total Hip Arthroplasty, Springer-Verlag: New York, 1999

[2] J. Gallo, P. Kaminek, V. Ticha, P. Rihakova, and R. Ditmar; "Theory of periprosthetic osteolysis: a review", Biomedical Papers, vol. 146, no. 2, pp. 21-28, 2002.

[3] L.A. Reynolds and E.M. Tansey, Eds, Early development of total hip replacement, Wellcome Witnesses to Twentieth Century Medicine, vol. 29. Wellcome Trust Centre for the History of Medicine at UCL: London 2007.

[4] Sargeant, and T. Goswami, Hip implants: Paper V. Physiological effects, Elsevier Materials and Design, vol. 27, pp. 287-307, 2006.

[5] S. Srimongkol, "A review of mathematical modelling in total hip replacement", International Mathematical Forum, vol. 7, no. 52, pp. 2561-2569, 2012.

[6] K.J. Saleh, I. Thongtrangan, and E.M. Schwarz, "Osteolysis: medical and surgical approaches", Clinical Orthopaedics and Related Research, no. 427, pp. 138-147, October 2004.

[7] D. Snyder, R. Chapell, W. Bruening, K. Schoelles, J. Kaczmarek, E. Kuserk, and E. Erinoff, "Horizon Scan on Hip Replacement Surgery", ECRI Evidence-Based Practice Center, Rockville, MD, Rep. 290-02-0019, December 22, 2006.

[8] S. Sivarasu, P. Beulah, and L. Mathew. "Optimization of skeletal hip implant cross-sections", In: National Conference on Computational Instrumentation-NCCI, April 19-20, 2010, pp. 37-43.

[9] C. Schmidler. Hip anatomy, function and common problems [Online]. Available: http://www.healthpages.org/anatomyfunction/hip-structure-function-common-problems/ , (2010, July 28).

[10] S. Pramanik1, A. K. Agarwal, and K. N. Rai, "Chronology of total hip joint replacement and materials development", Trends in Biomaterial and Artificial Organs, vol 19, no 1, pp.15-26, 2005.
[11] C.C. Harris, "Hip replacement prosthesis", U.S. Patent 6,248,132 B1, June 19, 2001.

[12] G.V. Subba Rao, and A.K. Goli, "Modular hip implant with shock absorption system", U.S. Patent 6,336,941 B1, January 8, 2002.

[13] S.G. Advani, M.H. Santare, F. Miller, and M. Joshi, "Stemless hip prosthesis", U.S. Patent 6,379,390 B1, April 30, 2002.

[14] B.J. Pope, J.K. Taylor, R.H. Dixon, C.F. Gardinier, L.M. Pope, D.C. Blackburn, M.A. Vail, and K.M. Jensen, "Prosthetic hip joint having at least one sintered polycrystalline diamond compact articulation surface", U.S. Patent 6,425,922 B1, July 30, 2002.

[15] S. Unger, "Femoral head calcar loading prosthesis", U.S. Patent 7, 641, 699 B2, January 5, 2010.

[16] J. Saladino, M.E. Nadzadi, and B. Burkinshaw, "Femoral head assembly with variable offset", U.S. Patent 7,833,277 B2, November 16, 2010.

[17] X. Li, P. Xie, and K.Y. Xie, "External proximal femoral prosthesis for total hip arthroplasty", U.S. Patent 7,931,691 B2, April 26, 2011

[18] H.D. Link, "Hip joint prosthesis with a shaft to be inserted into the femur", U.S. Patent 7,947,084 B2, May 24, 2011.

[19] K.S. Ely, A.C. Khandkar, R. Lakshminarayanan, and A.A. Hofmann, "Hip prosthesis with monoblock ceramic acetabular cup". U.S. Patent 8,133,284 B2, March 13, 2012.

[20] S. Tepic, "Wear-reducing geometry of articulations in total joint replacements", U.S. Patent 8,323,346 B2, December 4, 2012.

[21] M.A. Tuke, and A.C. Taylor, "Acetabular cup prosthesis and method of forming the prosthesis". U.S. Patent 8,372,155 B2, February 12, 2013.

[22] B. Walter, D. Brazil, and T. McTighe, "Neck sparing total hip implant system", U.S. Patent 8,470,049 B2, June 25, 2013.

[23] M-C. Ho Ba Tho, F. Roux, and M. Vanleene, "Acetabular prosthesis to be fixed without cement", U.S. Patent 8,506,644 B1, Aug13, 2013.

[24] R. Lambert, T. McLean, and D. Kelman, "Hip prostheses", U.S. Patent 8, 603, 182 B2, December 10, 2013.

[25] J.J. Shea, V.W. Shotton, and D.C. Kelman, "Universal liner", U.S. Patent 8, 679, 188 B2, March 25, 2014.

[26] J.S. Conway, R.L. Landon, and J.J. Shea, "Implant components and methods", U.S. Patent 8,700,198 B2, April 15, 2014.

Received: October 08, 2014

(c) Derar and Shahinpoor; Licensee Bentham Open.

This is an open access article licensed under the terms of the Creative Commons Attribution Non-Commercial License (http://creativecommons.org/licenses/by-nc/3.0/) which permits unrestricted, non-commercial use, distribution and reproduction in any medium, provided the work is properly cited. 